\title{
Adenine synthesis at Titan atmosphere analog by soft X-rays
}

\author{
Sergio Pilling ${ }^{1,2}$, Diana P. P. Andrade ${ }^{1,2}$, Alvaro C. Neto ${ }^{3}$, \\ Roberto Rittner ${ }^{3}$ and Arnaldo N. de Brito ${ }^{4}$ \\ ${ }^{1}$ Pontifícia Universidade Católica do Rio de Janeiro (PUC-Rio), 22453-900, \\ Rio de Janeiro, RJ, Brazil \\ ${ }^{2}$ Instituto de Pesquisa \& Desenvolvimento (IP\&D), Universidade do Vale do Paraíba \\ (UNIVAP), 12244-000, São Jose dos Campos, SP, Brazil \\ ${ }^{3}$ Universidade Estadual de Campinas (UNICAMP), 13084-971, Campinas, SP, Brazil \\ ${ }^{4}$ Laboratório Nacional de Luz Síncrotron (LNLS), 13083-970, Campinas, SP, Brazil
}

\begin{abstract}
In this work, we investigate the possible effects produced by soft X-rays (and secondary electrons) on Titan aerosol analogs in an attempt to simulate some prebiotic photochemistry. The experiments have been performed inside a high vacuum chamber coupled to the soft X-ray spectroscopy beamline at the Brazilian Synchrotron Light Source (LNLS). In-situ sample analysis were performed by a Fourier transform infrared spectrometer. The infrared spectra have presented several organic molecules, including nitriles and aromatic $\mathrm{CN}$ compounds. After the irradiation, the brownish-orange organic residue was analyzed ex-situ by gas chromatographic technique revealing the presence of adenine $\left(\mathrm{C}_{5} \mathrm{H}_{5} \mathrm{~N}_{5}\right)$, one of the constituents of the DNA molecule.
\end{abstract}

Keywords. astrochemistry, astrobiology, molecular processes, methods: laboratory, planets and satellites: Titan, Sun: X-rays

\section{Introduction}

Titan, the largest satellite of Saturn, has an atmosphere chiefly made up of $\mathrm{N}_{2}$ and $\mathrm{CH}_{4}$, and including many simple organic compounds. This atmosphere also partly consists of hazes and aerosols particles which shroud the surface of this satellite, giving it a reddish appearance. As a consequence of its high surface atmospheric pressure $(\sim 1.5$ bar) the incoming solar UV and soft X-ray photons are mostly absorbed allowing virtually no energetic photons to reach the surface. However, during the last 4.5 gigayears, the photolysed atmospheric molecules and aerosol particles have been deposited over the Titan surface composed by water-rich ice $(80-90 \mathrm{~K})$ delivered by comets. This process may have produced in some regions a ten meter size, or even higher, layers of organic polymer Griffith et al. (2003).

\section{Experimental methodology and results}

In this work investigate the chemical effects induced by soft X-rays in the Titan aerosol analog. The experiments have been performed inside a high vacuum chamber coupled to the soft X-ray spectroscopy (SXS) beamline at the Brazilian Synchrotron Light Source (LNLS), Campinas, Brazil. Briefly, a gas mixture simulating the titan atmosphere $\left(95 \% \mathrm{~N}_{2}, 5 \% \mathrm{CH}_{4}\right)$ was continuously deposited onto a polished $\mathrm{NaCl}$ substrate previously cooled at 13-14 $\mathrm{K}$ and exposed to synchrotron radiation (maximum flux between 0.5-3 keV) up to $73 \mathrm{hs}$. 
A small fraction of water and $\mathrm{CO}_{2}$ was also continuously deposited on the frozen substrate simulating thus, a possible heavy cometary delivery at Titan. The total energy deposited on the sample was about $\sim 10^{12} \mathrm{erg}$. In-situ sample analysis were performed by a Fourier transform infrared spectrometer (FTIR) during the irradiation and during the sample slowly heating to room temperature. The IR analysis has shown several organic molecules created and trapped in the ice, including the reactive cyanate ion $\mathrm{ONC}^{-}$, nitriles, and possibly amides and esters (Figure 1a).

After the irradiation, the brownish-orange organic residue (tholin) were analyzed exsitu by chromatographic (GC-MS) technique (Figure 1b) revealing the presence of adenine $\left(\mathrm{C}_{5} \mathrm{H}_{5} \mathrm{~N}_{5}\right)$, one of the constituents of DNA molecule. The complete description of the experimental setup and results can be found elsewhere Pilling et al. (2009).
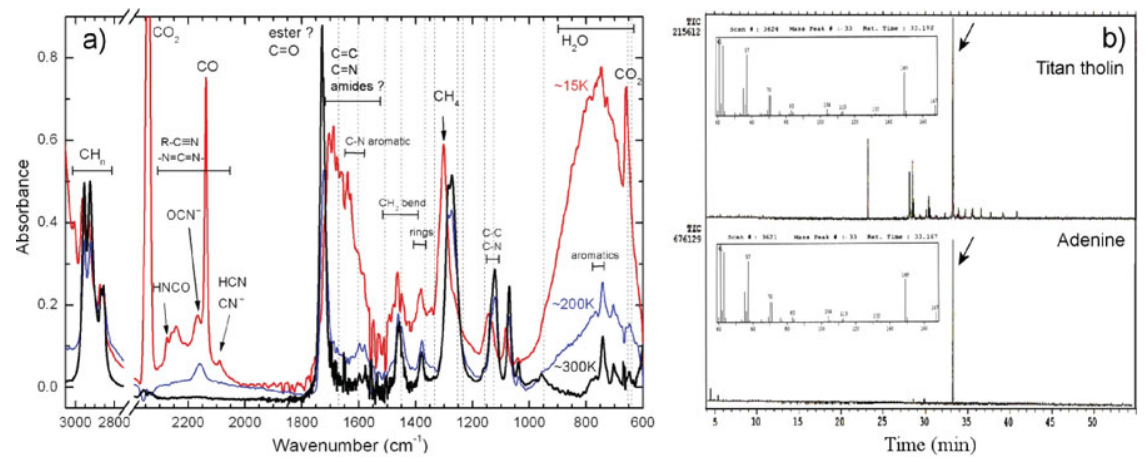

Figure 1. (a) Comparison between FTIR spectra of $73 \mathrm{~h}$ irradiated sample at 15,200 , and $300 \mathrm{~K}$. (b) Total-ion current chromatogram of processed the Titan tholin and of the adenine standard. Details can be found elsewhere (Pilling et al. (2009))

\section{Conclusions}

In this work, we present the chemical alteration produced by the interaction of soft $\mathrm{X}$-rays (and secondary electrons) on Titan aerosol analogs. The experiments simulate roughly $7 \times 10^{6}$ years of solar soft X-ray exposure on Titan atmosphere. Thermal heating of frozen tholin drastically changes its chemistry, resulting in an organic residue rich in $\mathrm{C}-\mathrm{C}$ and $\mathrm{C}-\mathrm{N}$ aromatic structures.

On Titan, the processed aerosols will be deposited along the time at the surface or at the bottom of lakes/rivers, leaving with them newly formed organic species. Gas chromatography analysis of the organic residue at room temperature has shown that among several nitrogen compounds, adenine, is one of the most abundant species produced due to irradiation by soft X-rays. This confirms previous studies suggesting that the organic chemistry in the Titan atmosphere and on the surface should be complex, being rich in prebiotic molecules such as adenine and amino acids (or its precursors species). Molecules such as these on the early Earth have found a place that allows life (as we know) to flourish, a place with liquid water.

\section{References}

Griffith, C. A., Owen, T., Geballe, T. R., Rayner, J., \& Rannou, P. 2003 Science, 300, 628

Pilling, S., Andrade, D. P. P., Neto, A. C., Rittner, R., \& de Brito, A. N. 2009 J. Phys. Chem. A 2009, In press, DOI:10.1021/jp902824v

Lappi, S. E. \& Franzen, S. 2004 Spectrochim. Acta, Part A, 60, 357 